\title{
Dynamic analysis of IgM and IgG antibody in asymptomatic patients as a more effective way to detect SARS-CoV-2 infection
}

\author{
Ping $\mathrm{Li}^{1}$, Ge Shen ${ }^{2}$, Zhenhua Zhu ${ }^{1}$, Shengjie Shi ${ }^{3}$, Yan $\mathrm{Hu}^{1}$, Ziyan Zeng ${ }^{2}$, Hui Zhou ${ }^{1}$, Qiong \\ $\mathrm{Li}^{1}$, Pan Zhu ${ }^{2}$, Gang Yang ${ }^{2}$, Zugui Liu ${ }^{2}$, Huiyuan $\mathrm{FU}^{2}$, Junyu $\mathrm{Hu}^{2}$, Ying $\mathrm{He}^{2}$, Qingting \\ Yang $^{2}$, Miao Dai ${ }^{2}$, Dan Zhou ${ }^{2}$, Qingqing $\mathrm{Lu}^{3}$, and Xiaobing Xie ${ }^{1}$ \\ ${ }^{1}$ First Hospital of Hunan University of Chinese Medicine \\ ${ }^{2}$ Loudi Center for Diseases Control and Prevention \\ ${ }^{3}$ Hunan University of Chinese Medicine
}

August 28, 2020

\begin{abstract}
Background:COVID-19 has become a global epidemic, close contacts and asymptomatic patients are worthy of attention. Methods: A total of 1844 people in close contact with 76 COVID-19 patients were investigated, and nasopharyngeal swabs and venous blood were collected for centralized medical quarantine observation. Real-time fluorescence used to detect 2019-nCoV nucleic acid in nasopharyngeal swabs of all close contacts, and colloidal gold method used to detect serum specific antibodies. Levels of IgM and IgG specific antibodies were detected quantitatively through chemiluminescence from the first nucleic acid negative date $(0 \mathrm{wk})$ within weekly periods of:1, 1-2, 2-3, and 6-7 weeks. Results:The total positive rate of the colloidal gold method $(88.5 \%, 23 / 26)$ was significantly higher $(\chi 2=59.182, \mathrm{P}<0.001)$ than that of the healthy control group $(2.0 \%, 1 / 50)$. There was significant difference in IgG concentration at different time points (0-7week) after nucleic acid turned negative $(\chi 2=14.034, \mathrm{P}=0.029)$. Serum IgG levels were significantly higher within weekly timepoints $4-5 \mathrm{wk}(\mathrm{Z}=-2.399, \mathrm{P}=0.016), 5-6$ $\mathrm{wk}(\mathrm{Z}=-2.049, \mathrm{P}=0.040)$, and $6-7 \mathrm{wk}(\mathrm{Z}=-2.197, \mathrm{P}=0.028)$, compared to $1-2 \mathrm{wk}$ after nucleic acid negative conversion. However, there was no significant difference $(\chi 2=4.936, \mathrm{P}=0.552)$ in IgM concentration between timepoints tested $(0-7$ weeks $)$ after nucleic acid conversion. The positive rates of IgM and IgG in asymptomatic patients $(\chi 2=84.660, \mathrm{P}<0.001)$ were significantly higher than the controls $(\chi 2=9.201, \mathrm{P}=0.002)$ within 7 weeks of nucleic acid negative conversion. Conclusions: The IgG concentration in asymptomatic cases remained at a high level after nucleic acid turned negative. Nucleic acid combined with IgM and IgG antibody detection is a more effective way to screen asymptomatic infections.
\end{abstract}

\section{Introduction}

In December 2019, an unexplained viral pneumonia broke out in Wuhan, Hubei Province, China ${ }^{[1,2]}$. The origin of the novel coronavirus remains unknown. However, gene sequencing analysis showed the virus was closely related to the coronavirus detected in wild animals. The novel coronavirus, named the severe acute respiratory syndrome coronavirus 2 (SARS-CoV-2) by the International Committee on Taxonomy of Viruses, causing the Corona Virus Disease 2019 (COVID-19), termed by the World Health Organization (WHO) ${ }^{[3]}$. COVID-19 has a lower mortality rate, is more contagious, and has caused a higher death toll than severe acute respiratory syndrome (SARS). These factors have led to a global pandemic within only a few months. At present, the epidemic in China has been well controlled due to a series of effective measures taken by the Chinese government, but it is still spreading abroad. The main challenge faced by the word in the future, in terms of the prevention and control of the epidemic, is the quick and accurate identification of the asymptomatic infection of SARS-CoV-2.

A positive result from a nucleic acid test is important for the diagnosis of asymptomatic patients with 
SARS-CoV-2 infection. Real-time fluorescence PCR (RT-PCR) is the main method to detect viral nucleic acid. However, the many limitations (i.e. long detection cycle, expensive reagents, and high requirements for sampling, technicians and laboratory conditions) can lead to high false negative rates and the untimely diagnosis and screening of asymptomatic cases. Therefore, there is an urgent need to explore a rapid, simple, and feasible method for the diagnosis and screening of COVID-19.

Serum immunoglobulin M (IgM) antibodies appear in the early stages of viral infection. This is followed by the production of serum immunoglobulin $\mathrm{G}(\mathrm{IgG})$ antibodies. These antibodies are essential for long-term immunity and immune memory. Detecting serum IgM and IgG antibodies may provide a valuable detection method for the diagnosis of COVID-19, especially when screening for asymptomatic infection. The aim of this study was to provide evidence for screening infection in asymptomatic patients with COVID-19 through the dynamic analysis of IgM and IgG levels.

\section{Materials and Methods}

\subsection{Patients}

In order to identify asymptomatic individuals, the Disease Control and Prevention (CDC) of Loudi conducted total of 1,844 close contacts (mean age 38.2 \pm 19.9 years old), including 1,003 males (mean age $38.7 \pm 19.9$ years old) and 841 females (mean age $37.7 \pm 20.0$ years) with 76 confirmed patients with COVID-19 from January to May 2020. Nasopharyngeal swabs were collected for SARS-CoV-2 nucleic acid detection from all close contacts. Participants then submitted to a 14-day medical quarantine observation period. Asymptomatic cases who received two consecutively negative nucleic acid tests after the expiration of medical quarantine were observed at home for an additional 14 days. Asymptomatic cases were defined by a positive result from SARS-CoV-2 nucleic acid detection of respiratory tract specimens or serum specific IgM antibodies without corresponding clinical symptoms of COVID-19 in close contacts, based on the diagnostic criteria for COVID-19 and the requirements of the treatment plan (trial version 6 and trial version 7 ) issued by the National Health Commission of the People's Republic of China and National Administration of Traditional Chinese Medicine ${ }^{[4,5]}$.

26 cases without clinical symptoms within 28 days of observation were recruited from close contacts following a positive SARS-CoV-2 nucleic acid detection. Close contacts whose nucleic acid test results were negative continued to be isolated for additional 14 days observation. 50 cases of healthy individuals from the First Hospital of Hunan University of Chinese Medicine served as the control group. Age and gender distribution did not differ between the study group and the control group $(\mathrm{p}>0.05)$. The details information of cases was presented in the results section. This study was approved by Ethics Committee of the First Hospital of Hunan University of Chinese Medicine.

\section{2 methods}

The SARS-CoV-2 nucleic acid extraction reagent (Magnetic bead method, Jiangsu Master Biotechnology Co., LTD. of China, item No: SDK60104) was used to extract viral nucleic acid with the aid of the automatic nucleic acid extraction instrument (Jiangsu Master Biotechnology Co., Ltd. of China, item No: SSNP-3000A). Open reading frame 1ab (ORF1ab) and nucleocapsid $(\mathrm{N})$ gene of dual nucleic acid detection kit was used for SARS-CoV-2 nucleic acid reverse transcription and amplification reagents (Shanghai Huirui Biotechnology Co., LTD. of China, product no.: vr-ii-120). Interpretation criteria: The value of cycle threshold (Ct) [?] 39.2 means the genes to be tested were negative; The $\mathrm{Ct}$ value $<35$ means the gene to be tested was positive; While the Ct value between 35 and 39.2, the results are in the grey area and the test should be repeated. Once repeated, if the $\mathrm{Ct}$ value [?] 39.2, the result was negative; however, if the $\mathrm{Ct}$ value $<39.2$ the result was positive. SARS-CoV-2 antibody detection kit (colloidal gold method, Guangzhou Wanfu Biotechnology Co., LTD. of China) and SARS-CoV-2 of IgM and IgG detection kit (chemiluminescence method, Shenzhen Ya Huilong Biotechnology Co., LTD. of China) were used for serum antibody assays and analyzed with a IFlash3000 automatic chemiluminescent immune analyzer (Shenzhen Ya Huilong biological technology Co., LTD. of China). Interpretation criteria: samples with IgM and IgG concentrations $<10.0 \mathrm{AU} / \mathrm{ml}$ are nonreactive (negative), while the concentrations [?]10.0 AU/ml are reactive (positive). All tests were conducted 
under manufacturing instructions for all of these kits.

\subsection{Detection of SARS-CoV-2 nucleic acid in close contacts}

Nasopharyngeal swabs were collected from 1844 close contacts and analyzed using a SARS-CoV-2 nucleic acid detection kit, following the manufacturer's instructions.

\subsection{Qualitative detection of serum SARS-CoV-2 antibodies in asymptomatic patients}

Blood was drawn from asymptomatic patients and centrifuged for 10 minutes to get serum. We detected SARS-CoV-2 antibody qualitatively with the colloidal gold method.

\subsection{Quantitative and dynamic detection of serum SARS-CoV-2 IgM and IgG specific antibodies in asymptomatic patients}

Pharyngeal swabs were collected for SARS-CoV-2 nucleic acid tests until tests were negative for 3 consecutive times. Blood was collected from the first nucleic acid negative date ( 0 week) within weekly periods of: 1 week, 1-2 week, 2-3 week, and 6-7 week. Blood was centrifuged at $3000 \mathrm{rpm}$ for 10 min. and sera were collected to detect levels of IgM and IgG specific antibodies quantitatively through chemiluminescence.

\subsection{Statistical Analysis}

Statistical analyses were performed using SPSS (v. 19.0) software with an $\alpha$ of 5\%. The measurement data are presented as mean \pm standard deviation or median (interquartile range [IQR] P25, P75). Comparisons between two groups were made using a T-test and Wilcoxon's rank sum test. Enumeration data are expressed as percentages using the $\chi 2$ test.

\section{Results}

\section{Epidemiology of close contacts}

Among the 1,844 close contact cases, the nucleic acid swabs for 33 individuals with no clinical symptoms came back positive; these individuals were treated as asymptomatic infected patients and placed under medical observation. One week later, 7 of them developed fever, cough, and other symptoms (Figure 1, table 1), confirming they had contracted COVID-19.

The rest 26 remaining cases with positive of nucleic acid were defined as asymptomatic cases, whose average age was $33.3 \pm 18.9$ years old, ranging from 3-77 years old. Age between the 15 males, averaging $34.2 \pm 17.6$ years old, and 11 females, averaging $32.1 \pm 21.4$ years old, there was no significant difference between genders ( $>0.05)$. Furthermore, there was no significant in age between 26 asymptomatic cases and the 7 patients with COVID-19 which was $29.7 \pm 17.5$ years old $(\mathrm{p}>0.05)$.

Time interval from nucleic acid in positive initially to the nucleic acid in negative for the first time was defined as the communicable period, which ranged from 1-31 days, and the median communicable period (MCP) was 7.5 days.

\section{Qualitative detection of antibodies to identify asymptomatic infections}

Blood was drawn from 26 asymptomatic infected cases for nucleic acid tests with positive results. 23 of the 26 asymptomatic patients were positive for COVID-19 specific antibodies by the colloidal gold method, only 3 were negative. The total positive rate of the colloidal gold method was $88.5 \%(23 / 26)$, which was significantly higher than that of the healthy control group $(2.0 \%)\left(\chi^{2}=59.182, \mathrm{p}<0.001\right)$. The positive rate between the male $(86.7 \%)$ and female $(90.9 \%)$ antibody in asymptomatic infection had no difference significantly $(\mathrm{p}>0.05)$.

\subsection{Quantitative dynamic changes in serum SARS-CoV-2 antibody in asymptomatic cases}

To further detect serum SARS-CoV-2 specific IgM and IgG antibodies, 17 cases screened from 26 asymptomatic patients to detect viral nucleic acid for several times with monitor on dynamic changes of antibodies. 
Among 17 cases of asymptomatic cases, male $(n=11)$ and female $(n=6)$ age ranged from 8-77 years old, averaging 31.5 years old (IQR 14.5-45.5). The comparison of SARS-CoV-2 antibody levels between groups at different time periods (0-7 week) after nucleic acid negative transformation showed as follows:

There was no significant difference in IgM concentration between time points tested (0-7 week) after nucleic acid conversion ( $p>0.05)$ (Figure 2a). However, a significant difference was found in IgG concentrations with time changed $(\chi 2=14.034, \mathrm{p}=0.029)$ (Figure $2 \mathrm{~b})$. The levels of serum IgM and IgG antibodies in different time periods (0-7 weeks) after nucleic acid negative conversion were showed in table 2.

Serum IgG levels were significantly higher within weekly time points $4-5$ week $(Z=-2.399, p=0.016)$, 56 week $(Z=-2.049, p=0.040)$, and 6-7 week $(Z=-2.197, p=0.028)$, respectively, compared to $1-2$ weeks after nucleic acid negative conversion. The serum IgG level within weeks $4-5$ of nucleic acid negative conversion was significantly higher than that of $3-4$ week $(Z=-2.038, \mathrm{p}=0.042)$.

There was no significant difference in serum IgG levels within 4 weeks, as well as exceeding the first month after nucleic acid negative transformation $(\mathrm{p}>0.05)$.

Among the 17 asymptomatic cases, 46 times of dynamic detection were performed on SARS-CoV-2 antibodies after the nucleic acid turned negative, including 10 times of positive results in IgM and 43 times of positive results in IgG (Figure 2). The positive detection frequencies of IgM and IgG were 21.7\% (10/46) and 93.5\% (43/46), respectively. Among the 50 healthy individuals, only one case was IgM positive with a concentration of $17.5 \mathrm{AU} / \mathrm{mL}$, and all IgG antibodies were negative. The positive detection rates of IgM and IgG in healthy individuals were $2.0 \%(1 / 50)$ and $0 \%(0 / 50)$, respectively.

The positive rates of IgM and IgG in asymptomatic group were significantly higher than that of the control group within 7 weeks of nucleic acid negative conversion, the statistics value was $\chi 2=84.660, \mathrm{p}<0.001$ and $\chi^{2}=9.201, \mathrm{p}=0.002$, respectively (Table 3 ).

\section{Discussion}

Cutting off the route of transmission is an important preventive measure for infectious disease. However, it has been reported recently that asymptomatic carrier can lead to person-to-person transmission in community due to the neglect. This will also pose a huge challenge for COVID-19 prevention and control. Therefore, the proportion of asymptomatic infections needs to be determined timely through proper laboratory techniques.

There are two situations in asymptomatic patients with COVID-19: infected individuals with positive viral nucleic acid test results but no recognizable symptoms and signs after observation for 14 days, and asymptomatic infections within the incubation period. As shown here, the nucleic acid test results of the infected individuals could be positive, even without self-perceived or clinically recognizable symptoms and signs during sampling, however, clinical manifestations could appear later.

It was reported that a 20-year-old female asymptomatic patient infected the five other members of her family after returning to Anyang, Henan province of China in January 10, $2020{ }^{[6]}$. The five family members developed symptoms (fever and cough) and were diagnosed with COVID-19. Nevertheless, the asymptomatic patient remains free of clinical symptoms, and her C-reactive protein and chest CT examination were normal. With the emergence of increased cases from abroad, the asymptomatic SARS-CoV-2 populations have attracted more attention as a hidden source of infection. As of August 21, 2020, there are 353 asymptomatic infections under medical observation in China, including 248 imported cases ${ }^{[7]}$.

Identifying asymptomatic infected populations early and accurately is essential for the epidemic prevention and control. Here, we provide evidence for screening infection in 26 asymptomatic individuals through dynamic analysis of IgM and IgG antibodies. It provides a laboratory basis for understanding the status of the immune system and the pattern of specific antibodies production. In this study, 33 asymptomatic cases with positive nucleic acid were screened, and 7 of them were converted to confirmed COVID-19 cases following their development of fever, cough, and chest CT imaging changes during the period of medical quarantine and observation, the remaining 26 remained asymptomatic. However, these 7 patients with 
COVID-19 did not receive continuous quantitative monitoring of serum antibody. So, it is impossible to compare and analyze the characteristics of antibody between asymptomatic and confirmed patients.

To further evaluate the production of SARS-CoV-2 specific antibodies in asymptomatic patients, blood specimens were collected regularly to detect the levels of serum SARS-CoV-2 specific IgM and IgG antibodies after nucleic acid converted to negative. The positive rate of IgM antibodies in asymptomatic individuals was significantly higher even when their nucleic acid converted to negative than that in healthy people. However, there was no significant difference in changes within 7 weeks after nucleic acid negative conversion $(\mathrm{p}<0.05)$. Our previous study showed that the positive rate of IgM in patients with COVID-19 was $75.9 \%$ [8]. These results suggest that IgM may be a useful target in screening the cases who previously infected by SARS-CoV-2 and healthy people. Furthermore, IgM in some asymptomatic individuals after nucleic acid convert to negative is not easy to degrade within 7 weeks.

According our study, the serum concentration of IgG in asymptomatic individuals after the nucleic acid converted to negative were in high level and increased with time in the 7 weeks we observed. The positive rate of serum IgG in asymptomatic group was $93.5 \%$ during 7 weeks after the nucleic acid turned to negative. While Long QX, et al ${ }^{[9]}$ reported that the positive rate of serum IgG in patients with acute phase COVID-19 was $18.9 \%$, while in convalescent-phase COVID-19 was $60.0 \%$, patients with acute phase.

To date, studies have focused more on antibodies in patients with a confirmed diagnosis rather than changes in antibody levels in asymptomatic patients following the nucleic acid negative conversion. For instance, Fei Xiang at al. found that the sensitivity and specificity of IgM were $77.3 \%$ and $100 \%$, respectively, and the sensitivity and specificity of $\operatorname{IgG}$ were $83.3 \%$ and $95.0 \%$, respectively, during detection of antibodies in COVID-19 patients ${ }^{[10]}$. Our previous study found that the sensitivity of IgG (90.5\%) was higher than that of $\operatorname{IgM}(75.9 \%)$, and the specificity of IgG (99.3\%) was higher than that of IgM (94.0\%) in COVID-19 patients [8]. Taken together, this indicates that the positive rate of IgM in asymptomatic patients after nucleic acid negative conversion is significantly lower than that in confirmed patients, while IgG remains at the same level as that of confirmed patients. Unfortunately, this study was unable to obtain serum specimens from asymptomatic patients when their nucleic acid was positive, and thus it was not possible to compare changes in antibody levels between nucleic acid positive and negative.

In conclusion, nucleic acid testing, though time consuming and susceptible to sampling errors, is recommended to be the main basis for the diagnosis of asymptomatic patients. Antibody detection holds great value in the diagnosis and identification of asymptomatic patients as it is fast, convenient, and the sampling is easily standardized.

\section{NOTES}

\section{Conflict of Interest}

The authors declare no conflicts of interest.

\section{Ethics approval}

This study was approved by the Ethics Committee of the First Hospital of Hunan University of Chinese Medicine.

\section{Funding}

This work was supported by the Innovative Provincial Construction Special Novel Coronavirus Pneumonia Emergency Response Rpecial Topic in Hunan Province Science and Technology Department 2020 (2020SK3009) and the First-class Discipline Open Fund Project of Hunan University of Chinese Medicine (2018YXJS0).

\section{References}

[1] Wang D, Hu B, Hu C, et al. Clinical characteristics of 138 hospitalized patients with 2019 novel coronavirus-infected pneumonia in Wuhan, China. JAMA, 2020 , 323(11): 1061-1069. 
[2] Li Q, Guan X, Wu P, et al. Early transmission dynamics in Wuhan, China, of novel coronavirus-infected pneumonia. N Engl J Med,2020, 382(13): 1199-1207.

[3] World Health Organization. WHO Director-General's remarks at the media briefing on 2019-nCoV on 11 February 2020. Available at:

https://www.who.int/zh/dg/speeches/detail/who-director-general-s-remarks-at-the-media-briefing-on-2019ncov-on-11-february-2020. Accessed 26 February 2020.

[4] National Health Commission of the People's Republic of China. Notice on the issuance of COVID-19 's diagnosis and treatment plan (version 6). Available at:http://www.nhc.gov.cn/yzygj/s7653p/202003/46c9294a7dfe4cef80dc7f5912eb1989.shtml.Accessed 03 March 2020.

[5] National Health Commission of the People's Republic of China. Notice on the issuance of COVID-19 's diagnosis and treatment plan (version 7). Available at:http://www.nhc.gov.cn/jkj/s3577/202003/4856d5b0458141fa9f376853224d41d7.shtml.Accessed 07 March 2020

[6] Bai Y, Yao L, Wei T, et al. Presumed Asymptomatic Carrier Transmission of COVID-19. JAMA, 2020, 323(14): 1406-1407.

[7] The latest situation of COVID-19 epidemic situation. National Health Commission of the People's Republic of China. Available at:http://www.nhc.gov.cn/. Accessed 21 August 2020

[8] Li Ping, Li Zhiyong Li, Zhao silin, et al. Preliminary study of serum 2019-nCoV IgM and IgG antibodies in the diagnosis of COVID-19. Clin J Lab Med, 2020, 4(43): 352-357.

[9] Long QX, Tang XJ, Shi QL, et al. Clinical and immunological assessment of asymptomatic SARS-CoV-2 infections. Nat Med, 2020, 26(8): 1200-1204.

[10] Xiang F, Wang X, He X, et al. Antibody Detection and Dynamic Characteristics in Patients with COVID-19 [published online ahead of print, 2020 Apr 19]. Clin Infect Dis, 2020, ciaa461. doi:10.1093/cid/ciaa461

\section{Figure Legends}

Figure 1. Epidemiological survey of close contacts with COVID-19.

Figure 2. Changes in serum 2019-nCoV IgM and IgG concentrations with time after 2019nCoV nucleic acid turned negative in 15 patients with asymptomatic novel coronavirus infection (Note: Abscissa W represents wk, red dotted line is the dividing line between negative and positive).

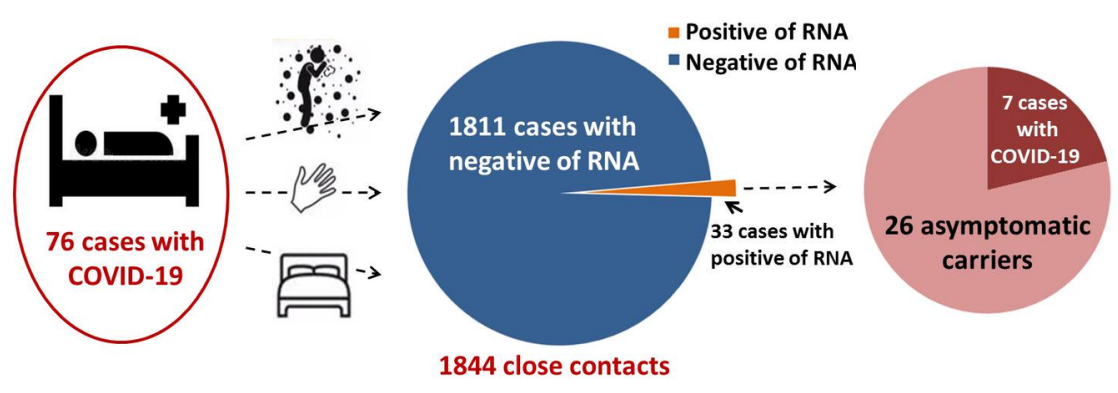



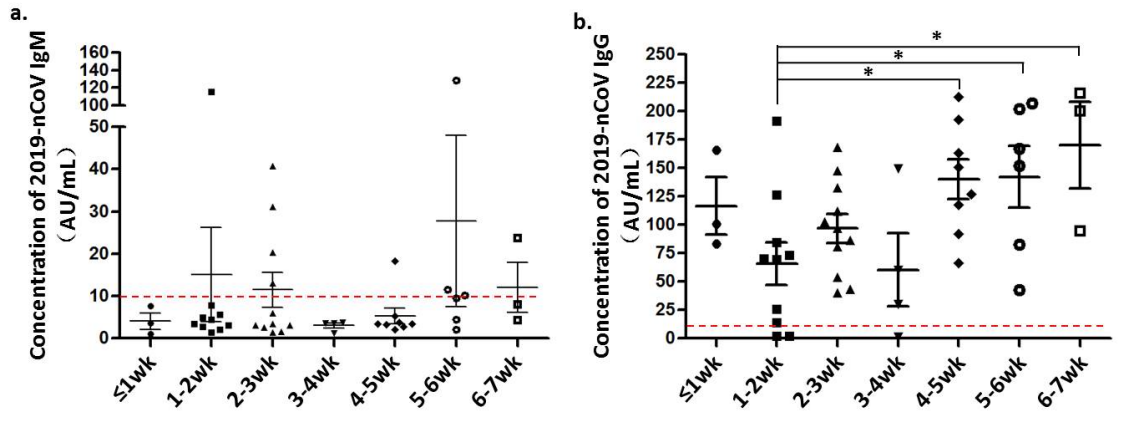

\section{Hosted file}

Table 1.docx available at https://authorea.com/users/353414/articles/477314-dynamic-analysisof-igm-and-igg-antibody-in-asymptomatic-patients-as-a-more-effective-way-to-detectsars-cov-2-infection

\section{Hosted file}

Table 2.docx available at https://authorea.com/users/353414/articles/477314-dynamic-analysisof-igm-and-igg-antibody-in-asymptomatic-patients-as-a-more-effective-way-to-detectsars-cov-2-infection

\section{Hosted file}

Table 3.docx available at https://authorea.com/users/353414/articles/477314-dynamic-analysisof-igm-and-igg-antibody-in-asymptomatic-patients-as-a-more-effective-way-to-detectsars-cov-2-infection 